\title{
Influence of interleukin $1 \beta$ on tenascin distribution in human normal and osteoarthritic cartilage: a quantitative immunohistochemical study
}

\author{
Xavier Chevalier, Pascal Claudepierre, Nicole Groult, Gaston-Jacques Godeau
}

\begin{abstract}
Objective - To determine the influence of IL-1 $\beta$ on the presence and the distribution of tenascin in matrix of human normal and osteoarthritic cartilage explants. Methods - Cartilage was grown in organotypic culture with or without IL-1 $\beta$ (10 $\mathrm{ng} \mathrm{ml}^{1}$ ). Tenascin antigen was detected on cryopreserved cartilage sections by immunohistochemical techniques with a monoclonal antibody directed against all tenascin isoforms (BC-4), and then quantified by video imaging densitometry.

Results - Tenascin was present in normal cartilage explants and increased in osteoarthritic cartilage explants. Treatment of normal and osteoarthritic cartilage explants with IL-1 $\beta\left(10 \mathrm{ng} \mathrm{ml}^{-1}\right)$ induced an increase in tenascin content, which was particularly high in normal cartilage and predominated in the superficial layers of damaged cartilage. There was no obvious correlation between proteoglycan loss and presence of tenascin.
\end{abstract}

Conclusions - In human normal and osteoarthritic cartilage explants, the presence and the distribution of tenascin are influenced by IL-1 $\beta$.

(Ann Rheum Dis 1996;55:772-775)

\section{Laboratoire de Biologie du tissu conjonctif, Faculté de médecine, the Department of Rheumatology of the Henri-Mondor hospital, Creteil, France \\ $\mathrm{X}$ Chevalier \\ P Claudepierre}

National Institute for Cancer Research, Genoa, Italy

N Groult

G J Godeau

Correspondence to: Dr X Chevalier, Department of Rheumatology, Henri-Mondor hospital, Bd De Lattre De Tassigny, 94010 Creteil, France.

Accepted for publication 6 June 1996
Interleukin-1 (IL-1) has been implicated in degradation of the extracellular matrix of cartilage during the osteoarthritic process through an increase in the expression of metalloproteinases. ${ }^{1}$ In osteoarthritis, a decrease in the content of normal cartilage components is associated with an accumulation of adhesive glycoproteins such as fibronectins ${ }^{2}$ and tenascin. $^{3}$ Tenascin is a large extracellular matrix glycoprotein $(1.900 \mathrm{kDa})$, comprising six similar subunits. ${ }^{5}$ Each subunit contains epidermal growth factor-like (EGF-L) repeats and Fn type III homology repeats, and a domain showing sequence similarities with fibrinogen. ${ }^{5}$ Tenascin has been isolated from various tissues undergoing remodelling. ${ }^{6-8}$

Factors that influence the accumulation of tenascin in osteoarthritic cartilage are unknown. The purpose of this study was to determine whether tenascin distribution in normal and osteoarthritic cartilage can be influenced by IL-1 $\beta$.

\section{Methods}

MATERIALS

All materials, unless otherwise specified were from Sigma (St Louis, MO, USA). OCT was from Miles (Napierville, IL, USA). Recombinant human IL1 $\beta$ was from Tebu Co (Paris, France). Alkaline phosphatase conjugated streptavidin-biotin complex was from Dako (Paris, France). Testicular hyaluronidase was from Serve (Heidelberg, Germany). Dishes for explant cultures were from Falcon (USA). Material for electrophoresis was from Biorad SA (Paris, France).

MONOCLONAL ANTIBODIES

A specific monoclonal (MAb) antibody (BC-4) against human tenascin was used to study the distribution of tenascin in cartilage. ${ }^{9}$

\section{HUMAN CARTILAGE EXPLANT CULTURE}

Cartilage tissues (from femoral head) were obtained from the Department of Orthopaedic Surgery, Hôpital Henri-Mondor (Creteil, France) at the time of joint replacement in patients with hip osteoarthritis $(n=10)$ (five females and five males, mean age: 70 , SD 4, years). Normal cartilage was obtained from three patients (femoral head cartilage at the time of joint replacement for femoral neck fracture) (two females and one male; mean age 72, SD 2, years). Each cartilage sample was immediately cut into small cartilage explants under aseptic conditions (25 mg average wet weight) and precautions were taken to select explants including deep to superficial layers. Normal and osteoarthritic cartilage explants were cultured in serum-free Iscove medium supplemented with bovine serum albumin, transferrin and lipid emulsion, and glutamine (2 $\mathrm{mM})$. Recombinant IL1 $\beta$ (10 $\mathrm{ng} \mathrm{ml}^{-1}$ ) was added to the medium and left for $24 \mathrm{~h}$. Media were then withdrawn and cartilage explants were frozen in liquid nitrogen, cryoprotected with OCT, and stored at $-80^{\circ} \mathrm{C}$ until used.

\section{IMMUNOHISTOCHEMISTRY}

Imunohistochemical localisation of tenascin was performed on $6 \mu \mathrm{m}$ frozen cartilage sections. Care was taken to orient cartilage 
slices during sectioning so as to obtain superficial, middle, and deep zones. Slides were previously coated with L-polylysine and fixed in acetone at $+4^{\circ} \mathrm{C}$ for $10 \mathrm{~min}$. Sections were pretreated with bovine testicular hyaluronidase $\left(0.5 \mathrm{mg} \mathrm{ml}^{1}: 1500 \mathrm{U} \mathrm{ml}^{-1}\right)$ at $37^{\circ} \mathrm{C}$ for $60 \mathrm{~min}$ in Tris- $\mathrm{NaCl}, \mathrm{pH} 5.8$, as this procedure has been shown to increase tenascin detection. To prevent non-specific binding of the primary antibody, sections were incubated with Tris-NaCL buffer containing 5\% BSA for 45 $\mathrm{min}$. Then anti-tenascin MAb (1:10) diluted in $5 \%$ bovine serum albumin was added for $1 \mathrm{~h}$. After washing in TBS buffer (Tris- $\mathrm{HCl} 0.05$ $\mathrm{M}, \mathrm{NaCL} 0.15 \mathrm{M}, \mathrm{pH} 7.4$ ), the slides were
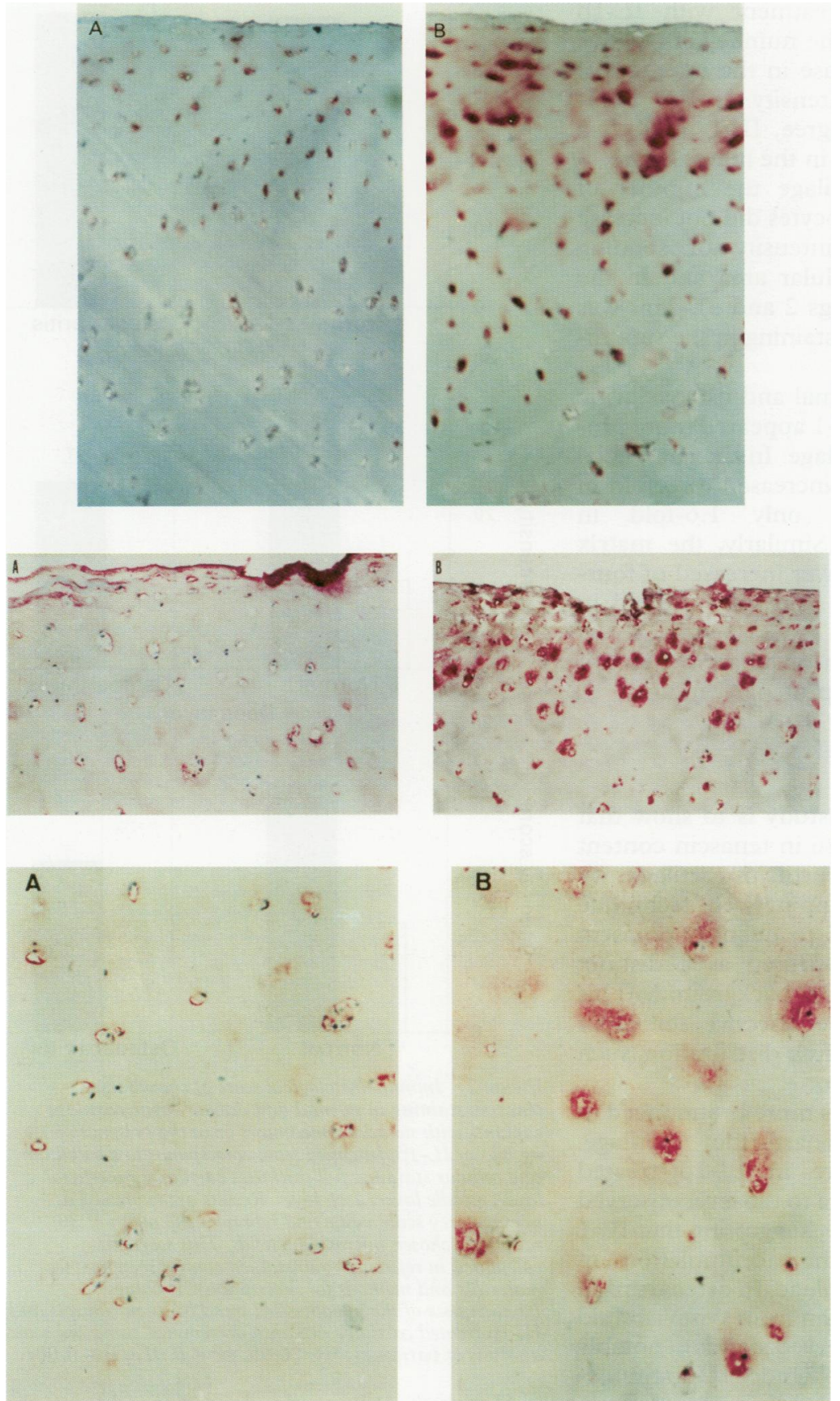

Figure 1 Presence of tenascin in normal and osteoarthritic cartilage explants. Immunohistological studies were performed using an avidin-biotin amplification system (see Methods). The monoclonal antibody (BC-4) used against tenascin recognises all isoforms of the molecule. Top: Normal cartilage. (A) Tenascin staining without added mediators $(\times 35)$. (B) Tenascin staining with IL-1 $\beta$ (10 $\left.\mathrm{ng} \mathrm{ml}^{-1}\right)$. Middle: Osteoarthritic cartilage. $(A)$ Tenascin staining without added mediators $(\times 35)$. (B) Tenascin staining with IL-1 1 (10 $\left.n g \mathrm{ml}^{1}\right)$ ( $\left.\times 35\right)$. Bottom: Osteoarthritic cartilage: magnification. (A) Tenascin staining

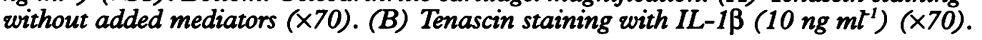

incubated with biotinylated goat anti-mouse immunoglobulin (diluted 1/1000) for $30 \mathrm{~min}$. Sections were then washed and incubated with a 1:50 dilution of soluble complex of alkaline phosphatase conjugated streptavidin (Dako kit), for $30 \mathrm{~min}$ at $22^{\circ} \mathrm{C}$. Immunoreactivity was revealed by addition of naphthol and fast-red solution. Levamisole was added to block endogenous alkaline phosphatase.

Appropriate controls were performed with another $\mathrm{MAb}$ from a hybridoma cell culture (anti- Ed-A fibronectin: IST-9), showing no staining in normal cartilage.

\section{AUTOMATED IMAGE ANALYSIS OF TENASCIN} STAINING

Immunopositivity for tenascin appears as a red to brown staining. The colourless background was averaged from one section to another, so that the sections could be compared. Computerised morphometric analysis of tenascin staining was carried out in the pericellular area (staining in and/or around the cells) and in the interterritorial matrix as previously described (Zeiss microscope, at $\times 40$ magnification, equipped with a Sophretec CF 126 PH R video camera).$^{10}$ Superficial, middle, and deep layers of the cartilage were tested. The volume of each area was automatically calculated by the computerised program and the number of cells was divided by the surface area. Each slide was analysed by two independent observers.

Data obtained were: (1) percentage of positive cells (cell score); (2) intensity of cell staining (intensity score); (3) the average matrix staining (matrix score).

DETECTION OF PROTEOGLYCAN

Proteoglycan staining was assayed by toluidine blue dye and compared with tenascin staining on the same sections.

\section{STATISTICAL ANALYSIS}

Significance of differences between groups was evaluated by one way analysis of variance (Bonferroni test) and was considered significant when $\mathrm{P}<0.05$.

\section{Results}

Immunohistolocalisation is shown in fig 1 . Scores and statistical analysis are summarised in figs 2 and 3.

PRESENCE OF TENASCIN IN NORMAL AND OSTEOARTHRITIC CARTILAGE EXPLANTS

In normal cartilage, tenascin was present mainly around the cells; in the interterritorial matrix it was very low (eightfold less when considering the full thickness of the cartilage) (fig 1). The staining was the most intense in the superficial layer while it was milder in the middle and deep layers.

In osteoarthritic cartilage, staining intensity in the pericellular area was 1.6-fold higher than in the interterritorial matrix when the full thickness of the cartilage is considered. Tenascin distribution in osteoarthritic cartilage 
showed some differences from normal cartilage (fig 1). First, the number of chondrocytes immunoreactive for tenascin was only slightly higher in osteoarthritic cartilage $(68 \%$ of positive cells in osteoarthritis $v 62 \%$ in normal cartilage). Second, the intensity (pericellular area) and the matrix scores were higher in osteoarthritic than in normal cartilage, whatever the layer (fig 1, fig 3). Third, in osteoarthritic cartilage there was intense staining on the cartilage surface in contact with synovial fluid.

PRESENCE OF TENASCIN IN NORMAL AND OSTEOARTHRITIC CARTILAGE EXPLANTS: INFLUENCE OF IL-1 $\beta$ (10 $\left.\mathrm{ng} \mathrm{ml}^{-1}\right)$

In normal cartilage, treatment with IL-1 $\beta$ induced an increase in the number of positive cells as well as an increase in the intensity of pericellular staining (intensity score). Similarly, but to a lesser degree, IL-1 induced a four- to fivefold increase in the matrix score.

In osteoarthritic cartilage the number of immunoreactive chondrocytes did not increase significantly, but the intensity of staining increased in the pericellular area and in the interterritorial matrix (figs 2 and 3). This was mostly due to enhanced staining in the superficial layer.

When comparing normal and osteoarthritic cartilage, the effect of IL-1 appeared more pronounced in normal cartilage. In the superficial layer the intensity score increased threefold in normal cartilage and only 1.6-fold in osteoarthritic cartilage. Similarly, the matrix score in the superficial layer increased of fourfold in normal cartilage and only twofold in osteoarthritic cartilage.

Otherwise distribution of tenascin was not strictly correlated to the proteoglycan loss.

\section{Discussion}

The main result of this study is to show that IL- $1 \beta$ induces an increase in tenascin content in both normal and osteoarthritis cartilage. We used an automated computerised technique which is a valid method to quantify tenascin because the staining appeared as a distinct structure on a colourless background. This technique would not be effective for other macromolecules with diffuse distribution, such as collagen or fibronectin.

The effect of IL-1 $\beta$ was more pronounced in normal than in osteoarthritic cartilage. Tenascin intensity score in IL-1 $\beta$ treated normal cartilage was close to the level observed in osteoarthritic cartilage, suggesting that IL-1 might contribute to the accumulation of tenascin in damaged cartilage. In osteoarthritic cartilage, tenascin diffusion from synovial fluid may account for the observed staining, notably on the cartilage surface. ${ }^{3}$ Under IL-1 stimulation there is an increased staining intensity in both the pericellular zone and its contingent interterritorial matrix, suggesting that the presence of tenascin in matrix distant from the chondrocyte may be due to a continuous increase in the production of tenascin by the chondrocyte.
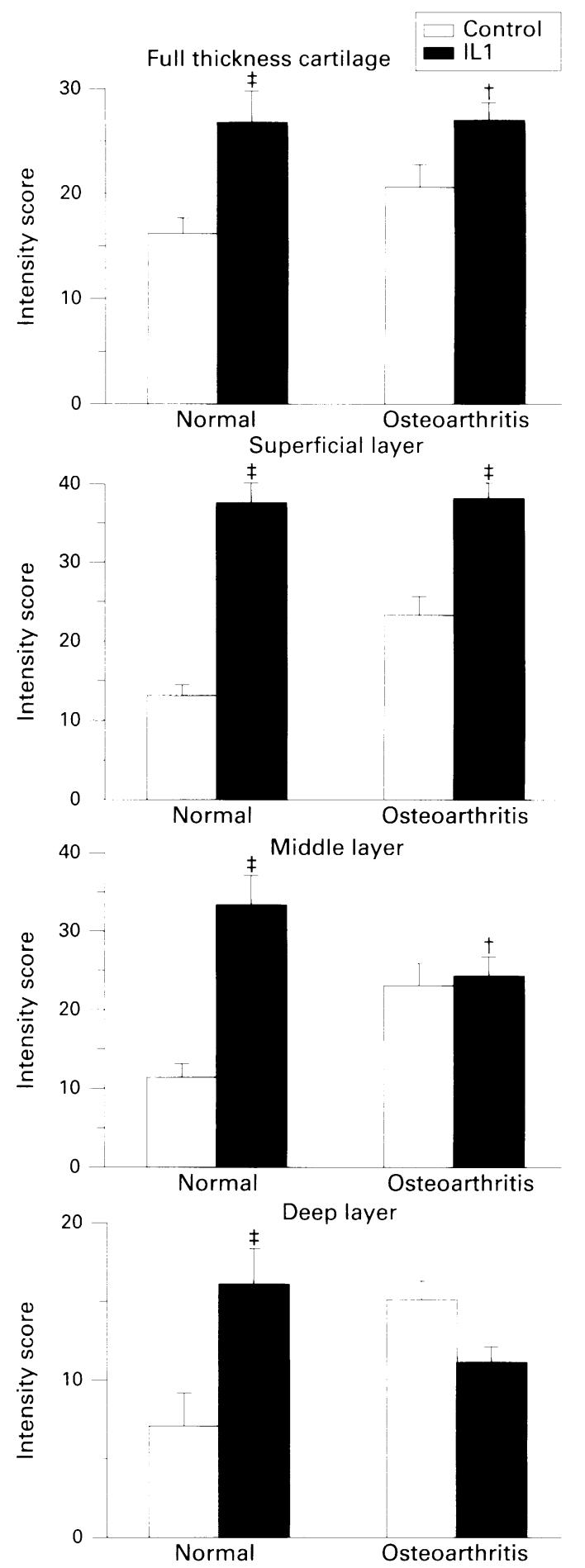

Figure 2 Immunohistological score of chondrocyte tenascin staining in normal and osteoarthritic cartilage explants with no added mediators or in the presence of 10 $n g t^{t}$ of $I L-1 \beta$. Intensity score corresponds to pericellular and cellular staining: full thickness cartilage; superficial layer; middle layer; deep layer. Results are expressed as average grey scale value and represent the mean of 30 randomly chosen microscopic fields. This score was calculated in reference to unstained control sections (see materials and methods for description). P values (significance of the hypothesised trend between controls and $I L-1 \beta$ treated cartilage explants, determined using one way analysis of variance): ${ }^{\star} P<0.05 ;+P<0.01 ; \neq P<0.001$.

However, this study does not address the mechanisms of tenascin accumulation. IL-1 can upregulate tenascin mRNA expression in synovitis. ${ }^{11}$ Alternatively, tenascin epitopes may become detectable during collagenase production or proteoglycan depletion induced by IL-1. 

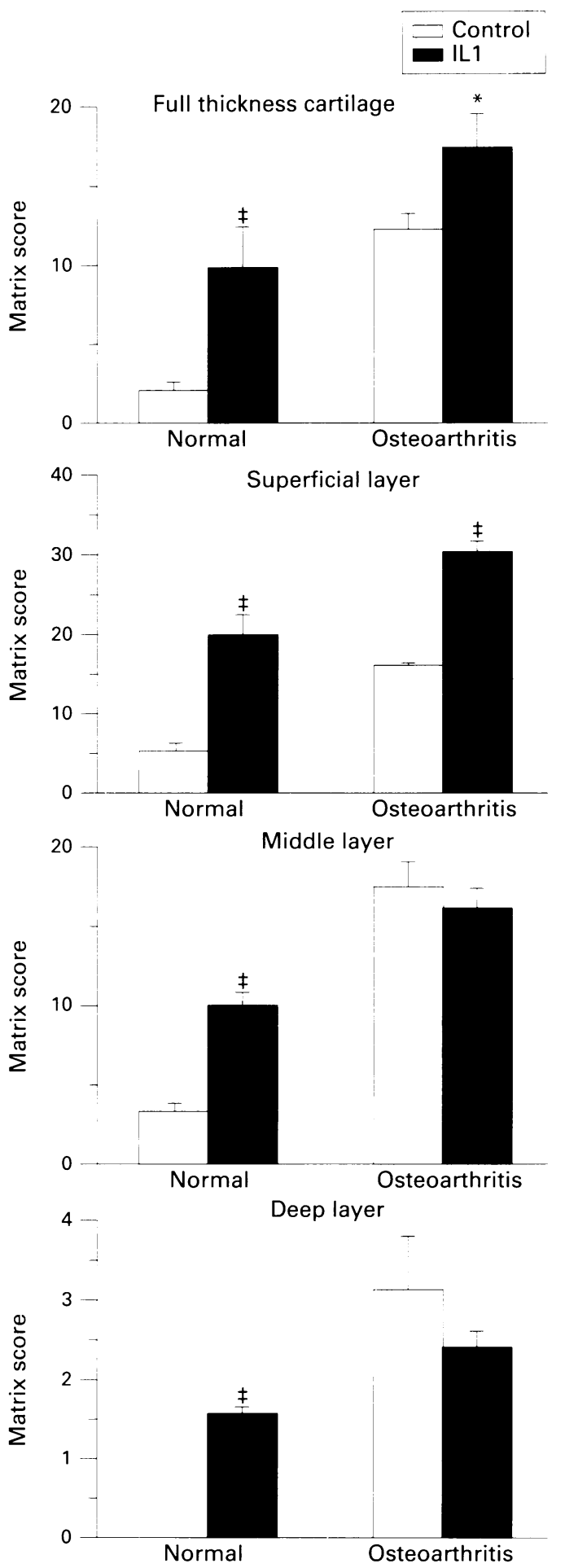

Figure 3 Immunohistological scores of tenascin staining in matrix distant from chondrocytes (matrix score) in normal and osteoarthritic cartilage explants with no added

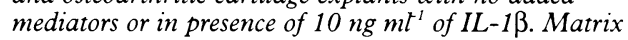
score corresponds to interterritorial matrix: full thickness cartilage; superficial layer; middle layer; deep layer. Results are expressed as average grey scale value and represent the mean average of 30 randomly chosen microscopic fields. This score was calculated in reference to unstained control sections (see Methods for description). P values (significance of the hypothesised trend between controls $I L-1 \beta$ treated cartilage explants, determined using one way analysis of variance): ${ }^{\star} P<0.05 ;+P<0.01 ; \neq P<0.001$.
Our results suggest that accumulation of tenascin may be part of the effects of IL-1 action, and do not imply that IL-1 is effective in vivo. Similarly IL-1 can modulate the production of other non-collagenous proteins such as thrombospondin. ${ }^{12}$

The functions of tenascin in damaged cartilage are not known. The presence of tenascin in normal cartilage suggests that it plays a physiological role. This protein has been implicated in the phenomenon of adhesion migration of cells. ${ }^{7}$ Because tenascin can impede cell adhesion to fibronectin substrate," ${ }_{14}$ it has been suggested that it might promote cell migration rather than cell adhesion. ${ }^{15}$ In osteoarthritic cartilage, tenascin might favour the migration of the chondrocytes towards the lesions, a hypothesis which fits well with its accumulation in the superficial layer.

We thank professor $\mathrm{L}$ Zardi $\mathrm{PhD}$, the National Institute for Cancer Research, Genoa, Italy, for providing the antibodies.

1 Gowen M, Wood DD, Ihrie EJ, Meats JE, Russel RGG. Stimulation by human interleukin- 1 of cartilage breakdown and production of collagenase and proteoglycanase by human chondrocytes but not by human osteoblasts in vitro Biochim Biophys Acta 1984;797:186--98.

2 Brown RA, Jones KL. The synthesis and accumulation of fibronectin by human articular cartilage. $\mathcal{F}$ Rheumatol 1990;17:65--72.

3 Salter DM. Tenascin in cartilage and synovium from arthritic knees. Br 7 Rheumatol 1993;32: 80--6.

4 Chevalier X, Groult N, Larget-Piet B, Zardi L, Hornebeck W. Tenascin distribution in articular cartilage from normal subjects and from patients with osteoarthritis and rheumatoid arthritis. Arthritis Rheum 1994;37:1013--22.

5 Erickson HP, Bourdon MA. Tenascin: an extracellular matrix protein prominent in specialized embryonic tissues and tumors. Annu Rev Cell Biol 1988;5:71--92.

6 Natali PG, Nicotra MR, Bigotti A, Bartolazze A, Motelese $\mathrm{M}$, Coscia $\mathrm{N}$, et al. Comparative analysis of the expression of extra-cellular matrix protein "tenascin" in normal human fetal, adult and tumor tissues. Int $f$ Cancer human fetal, adult

7 Mackie EJ, Halfter W, Liverani D. Induction of tenascin in healing wounds. F Cell Biol 1988;107:2757--67.

8 Koukoulis GK, Gould VE, Bhattacharya A, Gould JE, Howeedy AA, Virtanen I. Tenascin in normal, reactive, hyperplastic and neoplastic tissues: biologic and pathohyperplastic and neoplastic tissues: biologic and
logic implications. Hum Pathol 1991;22:636--43.

9 Siri A, Carnemolla B, Saginati M, Laprini A, Giorgio C, Barelle F, et al. Human tenascin: primary structure, pre-mRNA splicing patterns and localization of the epitopes recognized by two monoclonal antibodies. Nucleic Acid Res 1991;19:525--31.

10 Godeau GJ, Connord G, Jolivet O, Hornebeck W, Robert L. A selective histochemical method for the quantitative estimation of elastic fibers by computerized analysis. Anal mation of elastic fibers by comput
Quant Cytol Histol 1986;8:321--5.

11 McCachren SS, Lightner VA. Expression of human tenascin in synovitis and its regulation by interleukin-1. Arthriti Rheum 1992;35:1185--96.

12 Lyons-Giordano B, Kefalides NA, Brinker JM, Pratta MA, Arner EC. The effects of interleukin-1 on the expression of thrombospondin and fibronectin by rabbit articular chondrocytes. Exp Cell Res 1991;195:462--7.

13 Lotz MM, Bursdal CA, Erikson HP, McClay DR. Cell adhesion to fibronectin and tenascin: quantitative measurements of initial binding and subsequent strenthening response. $\mathcal{F}$ Cell Biol 1989;109:1795--805.

14 Murphy-Ulrich JC, Lightner VA, Aukhil I, Yan YZ, Erickson HP, Hook M. Focal adhesion integrity is down regulated by the alternative spliced domain of tenascin. $\mathcal{F}$ Cell Biol 1991;115:1127--36.

15 Mackie EJ, Tucker RP, Halfter W, Chiquet-Ehrismann R, Epperlein $\mathrm{HH}$. The distribution of tenascin coincides with pathway of neural crest cell migration. Development 1988 102:237--50. 